\title{
The segmental dynamics of a polymer electrolyte investigated by coherent quasielastic neutron scattering
}

\author{
P. Carlsson ${ }^{\text {a) }}$ \\ Department of Experimental Physics, Chalmers University of Technology, S-412 96 Göteborg, Sweden \\ R. Zorn \\ Institut für Festkörperforschung, Forschungszentrum Jülich, D-52425 Jülich, Germany \\ D. Andersson \\ Department of Experimental Physics, Chalmers University of Technology, S-412 96 Göteborg, Sweden \\ B. Farago \\ Institut Laue-Langevin, B. P. 156, F-38042 Grenoble Cedex, France \\ W. S. Howells \\ Rutherford Appleton Laboratory, Chilton, Didcot OX11 0QX, United Kingdom \\ L. Börjesson \\ Department of Applied Physics, Chalmers University of Technology, S-412 96 Göteborg, Sweden
}

(Received 29 September 2000; accepted 15 March 2001)

\begin{abstract}
The structural dynamics of a polymer electrolyte model material, poly(prolyene oxide) (PPO) $-\mathrm{LiClO}_{4}$ (and PPO for reference), has for the first time been studied using coherent quasielastic neutron scattering. By a combination of neutron spin echo and inverse time-of-flight techniques we investigate the relaxation function in an experimental time window $10^{-12} \leq t$ $\leq 10^{-8} \mathrm{~s}$ at a momentum transfer corresponding to the distance between neighboring interchain segments. We find that the relaxation of the correlation between neighboring chains is slower and more stretched in the polymer salt complex compared to the pure polymer. The data can, for both PPO and PPO- $\mathrm{LiClO}_{4}$, be described by a stretched exponential function with temperature independent stretching parameters. While the relaxation times follow the macroscopic viscosity for the former, they do not for the latter. The slower relaxation in PPO- $\mathrm{LiClO}_{4}$ compared to PPO and the failure of the viscosity scaling in $\mathrm{PPO}-\mathrm{LiClO}_{4}$ may be explained in terms of a temperature dependent effective molecular weight induced by cations acting as cross links between chains. We discuss the origin of the extra stretching of the relaxation in the polymer salt complex under the aspect of heterogeneity, comparing it with data in the literature. We find that the stretching to the major part is intrinsic or at most due to heterogeneities on an atomic length scale. The molecular length scale of the experiment allows for the first time a direct connection to the renewal time in the dynamic disordered hopping model for ion transport in polymer electrolytes. (C) 2001 American Institute of Physics. [DOI: 10.1063/1.1370073]
\end{abstract}

\section{INTRODUCTION}

Polymer electrolytes are materials of considerable interest for applications in electrochemical devices such as solid state batteries, since they yield a high energy density, are nontoxic, and have a significant ionic conductivity. Since the discovery that certain polymers can dissolve salts ${ }^{1}$ and function as solid electrolytes without any additional low molecular solvent, ${ }^{2}$ considerable insight has been gained and new improved materials have been synthesized. The knowledge about structural properties of the ion conducting amorphous phase $^{3}$ is, however, limited ${ }^{4-6}$ and the mechanism for the ionic conduction on the molecular level is an issue which is still far from settled. ${ }^{7}$ The mechanism must be fundamentally different from the mechanism in electrolytes with low molecular solvents, and the investigation of solvent free poly-

${ }^{a)}$ Electronic mail: f5xpc@fy.chalmers.se mer electrolytes is therefore also of considerable scientific interest. In this paper, we investigate a prototype system of an amorphous polymer electrolyte, poly(prolyene oxide) (PPO) complexed with $\mathrm{LiClO}_{4}$. We focus on the molecular dynamics of the polymer matrix, how it is modified by interaction with the ions and discuss the findings in relation to proposed models for the the ionic transport.

In the case of polyethers such as PPO and poly(ethylene oxide) (PEO), the cations are solvated by the ether oxygens, and several different structural arrangements which also may involve cation-anion coordinations are possible. ${ }^{6}$ The interaction between the polymer chains and the anions is weak. ${ }^{6}$ Structural studies of crystalline PEO complexed with salt ${ }^{8,9}$ have contributed valuable insights into the coordination of the cations to the ether oxygens, but are of limited value for predictions of properties of the amorphous phase where the ion conduction occurs. Neutron diffraction experiments on PEO-LiI melts ${ }^{10}$ in combination with molecular dynamics 
simulations ${ }^{11}$ indicate that in the melt the lithium to etheroxygen coordination number is larger than in corresponding crystalline complexes and decreases with increasing temperature. Spectroscopic studies ${ }^{4,12-15}$ have shown that the solvation reaction is complex and not only dissociated ions but also associated ion species, e.g., ion pairs, triplets, and clusters whose abundances increase with salt concentration are found. ${ }^{16}$

In general, the ionic conductivity first increases with salt concentration and then at higher concentrations decreases due to several effects. The formation of associated ion species leads to a reduction of the number of available dissociated ions ${ }^{13,14}$ and the molar conductivity decreases. The major effect which limits the ionic conductivity at high salt concentrations is, however, the decrease in the charge carrier mobility. There are strong indications that the mobility of ions is promoted by the segmental motion of the polymer chains ${ }^{17-19}$ and the ionic transport is therefore believed to be largely determined by the dynamics of the polymer host. ${ }^{20}$ The interaction between the polymer chains and the ions leads, however, to a slowing down of the segmental motion which may explain the decrease in conductivity at high salt concentrations. ${ }^{18}$ A microscopic description of the motion of the ions in the polymer host is offered by the dynamic bond percolation (DBP) model, ${ }^{21,22}$ and the more general but also less detailed dynamic disordered hopping (DDH) model. ${ }^{23,24}$ When applying these models to polymer electrolytes, an ion is considered to perform a motion which is confined to a small region in the polymer host and is characterized by a time scale $\tau_{h}$. The polymer matrix rearranges on a (usually longer) time scale $\tau_{R}$ which allows the ion to escape the confinement.

In the present work, we investigate the segmental dynamics in a polymer electrolyte model material using the neutron spin echo (NSE) technique in combination with time-of-flight (TOF) neutron scattering. ${ }^{25} \mathrm{We}$ thereby obtain the relaxation function in a time range from approximately $10^{-12}-10^{-8}$ at a wave vector corresponding to the the distance between the polymer chains. With this data it is possible for the first time to use the DDH model in conjunction with a microscopically derived renewal time $\tau_{R}$.

\section{EXPERIMENT}

In this work, the same samples of PPO and PPO- $\mathrm{LiClO}_{4}$ of concentration $\mathrm{O}: \mathrm{Li}=16: 1$ were investigated at the IN11 neutron spin echo spectrometer, Institut Laue-Langevin, France, and the high resolution backscattering neutron spectrometer IRIS at the ISIS pulsed spallation source, Rutherford-Appleton Laboratory, UK. The instruments were chosen to obtain a wide experimental time window, $\sim 10^{-12}-10^{-8} \mathrm{~s}$, since the relaxation function for the $\alpha$ process in polymer electrolytes is stretched over several decades and shows a strong temperature dependence. In order to reduce the incoherent scattering deuterated polymers were used. Both instruments were set up to focus on the $Q$ value of $1.45 \AA^{-1}$, since this is close to the first sharp diffraction peak (FSDP) of the static structure factors of PPO and PPO- $\mathrm{LiClO}_{4} \mathrm{O}: \mathrm{Li}=16: 1 .^{26,27}$

\section{A. Sample preparation}

The samples were prepared from freeze dried deuterated PPO with hydroxy end groups $\left(\mathrm{OH}-\left[\mathrm{CD}_{2}-\mathrm{CD}\left(\mathrm{CD}_{3}\right)-\mathrm{O}\right]_{N}-\mathrm{H}\right)$ (Polymer Source, Inc.) and vacuum dried $\mathrm{LiClO}_{4}$. The molecular weight stated by the supplier was $M_{n}=3700$ but a subsequent gel-permeation chromatography supports a significantly lower value, $M_{n}$ $\approx 2000{ }^{28,29}$ The salt was dissolved in the polymer under stirring using minute amounts of dry acetonitrile as intermediate solvent. The solution, and a sample of pure PPO were freeze dried on a vacuum line $(P \sim 1 \mathrm{mPa})$ until no bubbles evolved, and then transferred to sample containers in a dry argon atmosphere glove box. There were no signs of any residual acetonitrile in similar hydrogenous samples, produced as above, when characterized by Raman spectroscopy. ${ }^{30}$

Characterization of the samples with nuclear magnetic resonance (NMR) showed that only $86 \%$ of the hydrogen atoms attached to the polymer chain backbone were replaced by deuterium but the methyl groups were nearly completely deuterated $(>99 \%) .{ }^{29}$ The overall degree of deuteration of the polymer was found to be approximately $90.2 \%$ by measuring the scattering cross section in neutron diffraction experiments. ${ }^{27}$ For the PPO sample the coherent and incoherent scattering cross section are $\sigma_{\text {coh }}=4.46$ and $\sigma_{\text {incoh }}$ $=6.56 \mathrm{~b} /$ atom and the absorption is $\sigma_{\mathrm{abs}}=0.09 \mathrm{~b} /$ atom at $\lambda$ $=7.5 \AA$, the wavelength used on IN11. The sample of PPO- $-\mathrm{LiClO}_{4} 16: 1$ has the cross sections $\sigma_{\text {coh }}=5.93$ and $\sigma_{\text {incoh }}=4.90 \mathrm{~b} /$ atom and an absorption of $\sigma_{\text {abs }}=2.70 \mathrm{~b}$ at $\lambda$ $=7.5 \AA$.

\section{B. IN11 neutron spin echo experiment}

In the experiment on IN11, a wavelength $\lambda=7.5 \AA$ (1.45 $\mathrm{meV}$ ) and a scattering angle of $20^{\circ}$ were chosen to obtain a $Q$ value of $1.45 \AA^{-1}$. The distribution of the wavelength was approximately triangular in shape with a width of $15 \%$ full width at half maximum (FWHM). The cutoff of the analyzer mirrors was at $\lambda=3.5 \AA(6.7 \mathrm{meV})$. This corresponds to a bandpass $-1.45 \mathrm{meV}<E<5.2 \mathrm{meV}$ of the energy transfers accepted by the instrument. In an ideal NSE experiment the quantity $S(Q, t) / S(Q, t=0)$ is measured directly in the time domain. Due to the bandpass on the analyzed neutron energies the normalization is not strictly to $t=0$ but rather to the Fourier time corresponding to the width of the bandpass, approximately $1 \mathrm{ps}$. Incoherent scattering is suppressed by a factor of $1 / 3$ because it implies a spin flip with probability $2 / 3$. For details on the NSE experimental technique, see for instance Ref. 31. The effects due to multiple scattering and absorption are small for the NSE technique, therefore no such corrections were performed.

The PPO sample was investigated at temperatures $T$ $=15,220,240,260,280,290,300,310,325$, and $334 \mathrm{~K}$, and the $\mathrm{PPO}-\mathrm{LiClO}_{4}$ sample of concentration $\mathrm{O}: \mathrm{Li}=16: 1$ was investigated at $T=260,280,290,300,310,325$, and $334 \mathrm{~K}$. The sample of PPO measured at $\sim 15 \mathrm{~K}$ was used as resolution function. The containers were airtight flat aluminum vessels with sample thickness of $2.0 \mathrm{~mm}$. 


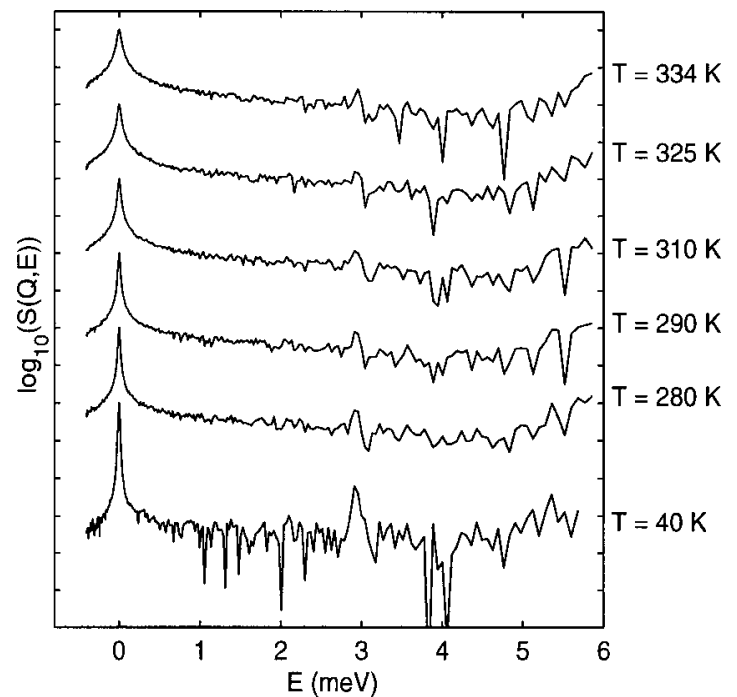

FIG. 1. The scattering function $S(Q, \omega)$ averaged to constant $Q$ $=1.45 \AA^{-1} \pm 7.5 \%$ vs energy transfer $E=\hbar \omega$ for PPO.

\section{IRIS TOF experiment}

The inverse geometry TOF instrument IRIS ${ }^{32}$ was used with the pyrolythic graphite 002 (PG002) analyzer reflection at $E_{1}=1.85 \mathrm{meV}$ with an energy resolution of $15 \mu \mathrm{eV}$ (FWHM). The accessible momentum transfer range in this setup is $0.5<Q<1.8 \AA^{-1}$ (elastic). The choppers were set to cover the energy transfer range $-0.4<E<6 \mathrm{meV}$. Because of the high energy transfers to be detected a berryllium filter was used to suppress neutrons passing the analyzer via the PG004 reflection.

The samples of PPO and PPO- $\mathrm{LiClO}_{4}$ of concentration $\mathrm{O}: \mathrm{Li}=16: 1$ were investigated at temperatures $T=40,280$, $290,310,325$, and $334 \mathrm{~K}$. The sample containers were cylindrical in shape with an outer diameter of $23 \mathrm{~mm}$ and a sample thickness of $0.5 \mathrm{~mm}$. Conversion to the scattering function $S(Q, \omega)$ and absorption corrections were performed using standard site software. ${ }^{33}$

Because of the high energy range it is important to consider that a spectrum registered by a detector at a certain angle contains scattering with a momentum transfer $Q$ which varies with the energy transfer $E$. In order to obtain a situation corresponding to that of IN11, for each energy transfer channel the detectors containing data with $Q=1.45 \AA^{-1}$ $\pm 7.5 \%$ were averaged to "constant $Q$ " spectra which were subsequently used in the Fourier transform. Figures 1 and 2 show the spectra for PPO and PPO- $\mathrm{LiClO}_{4} 16: 1$ when averaged to constant $Q=1.45 \AA^{-1}$. All spectra show a spurious inelastic peak at about $2.8 \mathrm{meV}$. This peak is present in all detectors at the same energy transfer and decreases with temperature and angle. Furthermore there is an unphysical increase of $S(Q, \omega)$ at high energy transfers $E>4 \mathrm{meV}$ which may be due to an incomplete operation of the beryllium filter. Because the origin of both features is not fully understood we refrained from devising any correction but used the spectra only up to $E_{\max }=2.5 \mathrm{meV}$.

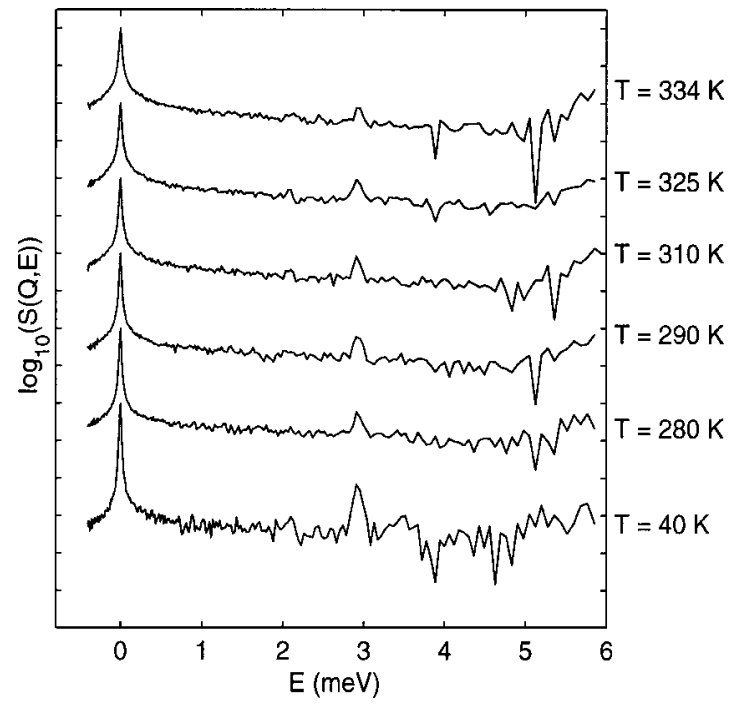

FIG. 2. The scattering function $S(Q, \omega)$ averaged to constant $Q$ $=1.45 \AA^{-1} \pm 7.5 \%$ vs energy transfer $E=\hbar \omega$ for PPO- $\mathrm{LiClO}_{4} 16: 1$.

\section{Fourier transform of IRIS data}

The Fourier transform has been done by directly applying the discrete complex Fourier integral

$$
S(Q, t)=\sum_{k=1}^{N} S\left(Q, \omega_{k}\right) \exp \left(-i \omega_{k} t\right) \Delta \omega_{k}
$$

to the constant $Q$ spectra; $N$ denoting the number of time-offlight channels, $\omega_{k}$ the angular frequency, and $\Delta \omega_{k}$ the width of channel $k$. The data were not subjected to "binning" or interpolation on the energy scale before the numerical Fourier transform. Because of the spurious peak at $2.8 \mathrm{meV}$ and the increasing background at high energy transfers, only the part of the spectra up to $\hbar \omega_{\max }=2.5 \mathrm{meV}$ was used in the transform. Since energy transfers in the range $-2.5<E$ $<-0.05 \mathrm{meV}$ (with respect to the sample) are not accessible with IRIS or have large errors, the spectra on the energy gain side were mirrored to cover this range employing the detailed balance principle. The Fourier times were chosen as multiples of $\pi / \omega_{\max }=0.8 \mathrm{ps}$ in order to minimize "wiggles" resulting from the cutoff.

The effect of the convolution with the resolution function of the instrument reduces to a multiplication in time domain. Therefore it can be easily removed by dividing the Fourier transformed spectra by a Fourier transform of the resolution function. For the latter, the spectra at $T \approx 40 \mathrm{~K}$ of the same sample were used since they were found to contain negligible quasielastic scattering. The quotient may have an imaginary part due to an offset in the elastic line position. Therefore the absolute value was taken which is equivalent to shifting the elastic line to zero energy transfer in frequency domain.

This division implies a normalization to the low temperature structure factor, $S(Q, t) / S_{T=40 \mathrm{~K}}(Q)$. Because a clear change of the position of the structure factor peak could be observed when the samples were heated to the measurement temperatures near $300 \mathrm{~K}$ this normalization is not very meaningful. We therefore renormalized the data to the inte- 


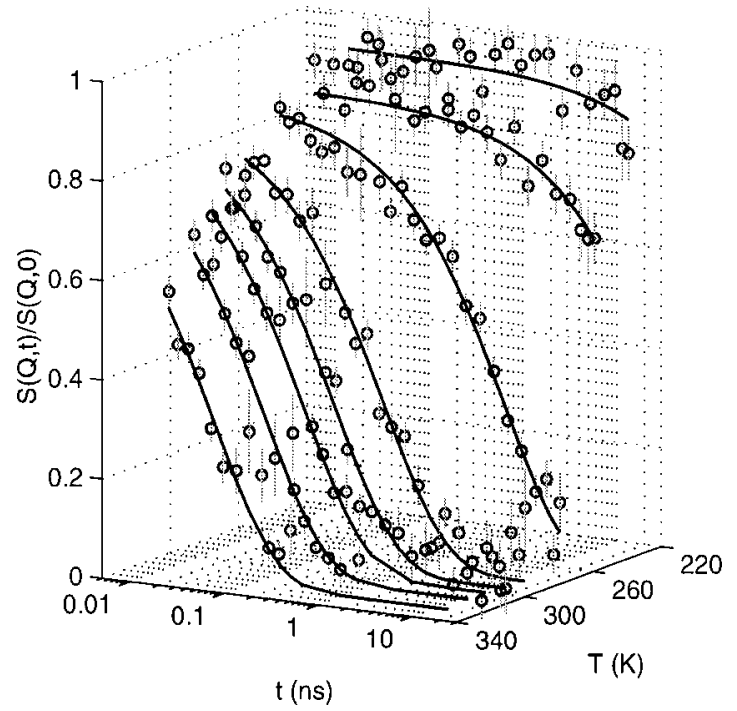

FIG. 3. $S(Q, t) / S(Q, 0)$ at $Q=1.45 \AA^{-1}$ for PPO as obtained from the NSE experiment (rings and error bars) and fits of the KWW, Eq. (2) (continuous lines) performed as described in the text.

grals over the energy range $-2.5 \mathrm{meV}<E<2.5 \mathrm{meV}$. Thereby, the resulting quantity is $S(Q, t) / S(Q, t=0)$ in the same sense as for the NSE experiment but with a slightly different energy band used.

\section{RESULTS}

Figures 3 and 4 present the $S(Q, t) / S(Q, 0)$ at $Q$ $=1.45 \AA^{-1}$ for PPO and PPO- $\mathrm{LiClO}_{4} \mathrm{O}: \mathrm{Li}=16: 1$ as obtained from the NSE experiment. It is seen, directly from the experimental data, that the relaxation behavior of the polymer salt system is slower and more stretched compared to that of the pure polymer.

Figures 5 and 6 present $S(Q, t) / S(Q, 0)$ at $Q$ $=1.45 \AA^{-1}$ for PPO and PPO- $\mathrm{LiClO}_{4} \mathrm{O}: \mathrm{Li}=16: 1$, calculated from the IRIS spectra in Figs. 1 and 2 as described in

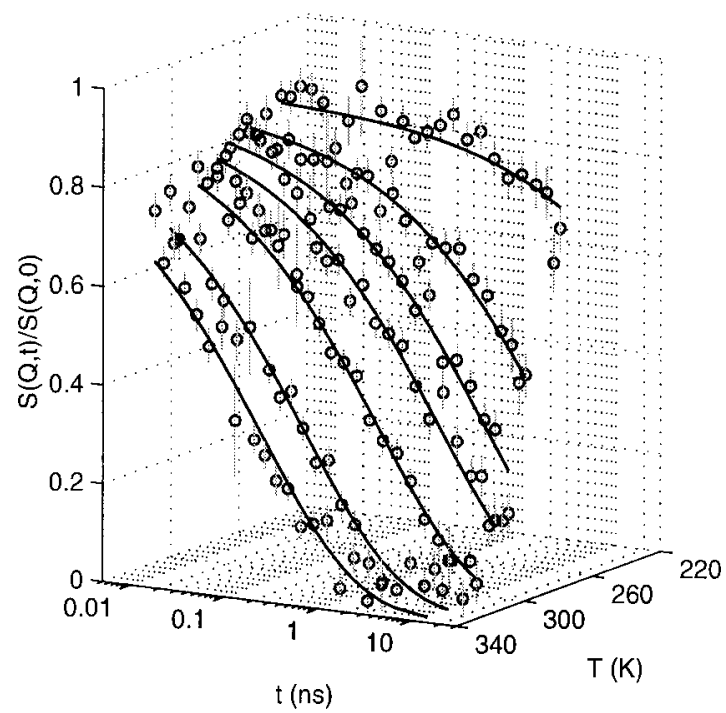

FIG. 4. $S(Q, t) / S(Q, 0)$ at $Q=1.45 \AA^{-1}$ for $\mathrm{PPO}-\mathrm{LiClO}_{4} \mathrm{O}: \mathrm{Li}=16: 1$ as obtained from the NSE experiment (rings and error bars) and fits of the KWW, Eq. (2) (continuous lines) performed as described in the text.

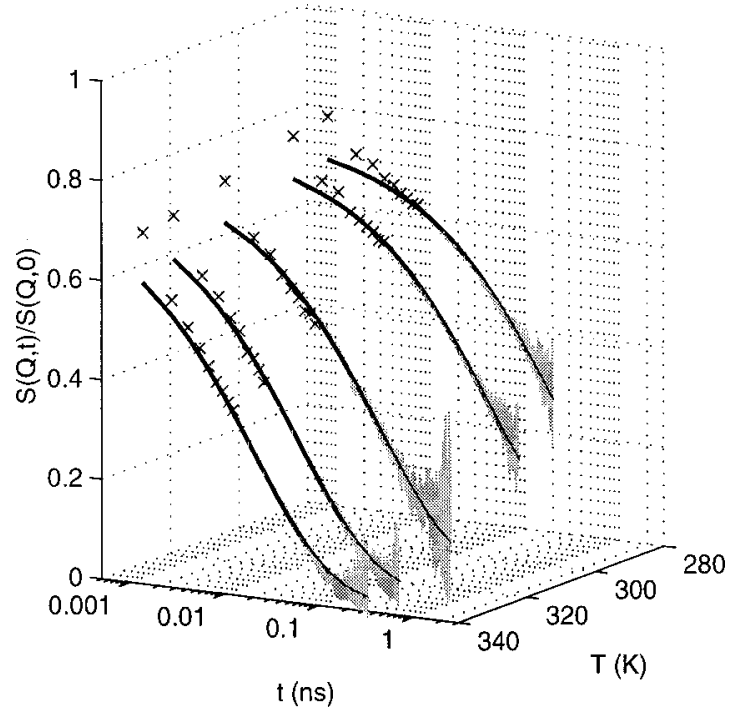

FIG. 5. $S(Q, t) / S(Q, 0)$ at $Q=1.45 \AA^{-1}$ for PPO as obtained by Fourier transform from the IRIS experiment and fits of the KWW, Eq. (2) (continuous lines) performed as described in the text. Because of the large number of data points only those for $t<8$ ps have been marked by crosses and for the longer times only their error bars are shown.

Sec. II D. Also from these data the general result of slower relaxation and more pronounced stretching can be seen.

In a first analysis, a stretched exponential [KohlrauschWilliams-Watts (KWW)] function

$$
\frac{S(Q, t)}{S(Q, 0)}=A \exp \left(-\left(t / \tau_{K}\right)^{\beta}\right),
$$

where $A$ is the amplitude, $\beta$ the stretching parameter, and $\tau_{K}$ the characteristic time of the relaxation, was fitted to the data. The fits are shown as continuous lines in Figs. 3-6 and it is seen that the function describes the relaxations in both materials well. In these fits, unique $A$ and $\beta$ parameters were

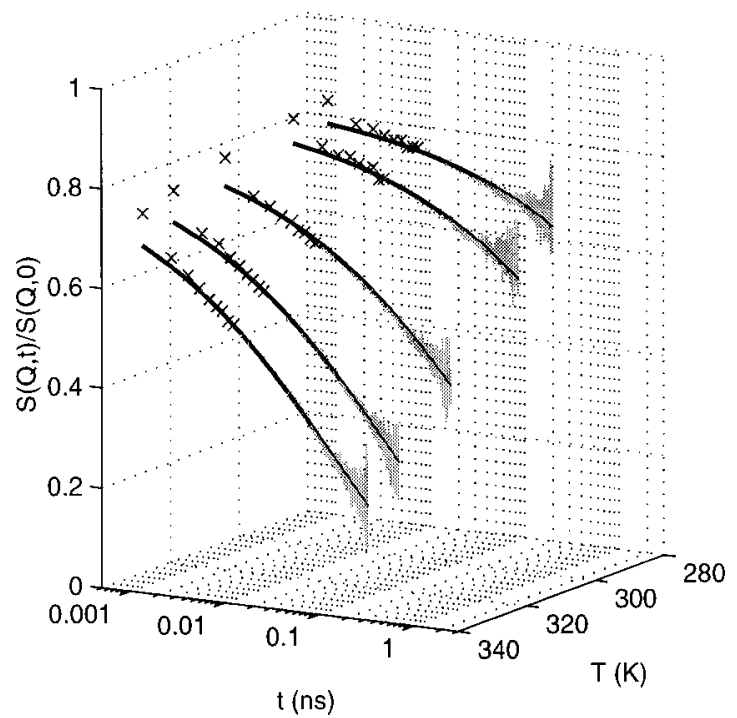

FIG. 6. $S(Q, t) / S(Q, 0)$ at $Q=1.45 \AA^{-1}$ for $\mathrm{PPO}-\mathrm{LiClO}_{4} \mathrm{O}: \mathrm{Li}=16: 1$ as obtained by Fourier transform from the IRIS experiment and fits of the KWW, Eq. (2) (continuous lines) performed as described in the text. Because of the large number of data points only those for $t<8$ ps have been marked by crosses and for the longer times only their error bars are shown. 
TABLE I. The values of the common parameters found by fitting of the KWW function [Eq. (2)] to the data obtained for the four sample/instrument combinations. The standard deviations estimated as described in the text are smaller than $2 \%$, though with possible systematic errors included, we estimate that these parameters are subject to an uncertainty of about $5 \%$.

\begin{tabular}{lccc}
\hline \hline Sample & Inst. & $A$ & $\beta$ \\
\hline PPO & IN11 & $0.86^{\mathrm{a}}$ & 0.67 \\
PPO & IRIS & 0.73 & 0.56 \\
PPO-LiClO & IN11 & 0.89 & 0.45 \\
O:Li=16:1 & IRIS & 0.81 & 0.37 \\
\hline \hline
\end{tabular}

${ }^{\mathrm{a}} A=0.93$ for the spectrum at $T=220$.

used for each of the four instrument/sample combinations, while the characteristic $\tau_{K}$ was fitted individually for each spectrum, the only exception being the spectra of PPO at $T$ $=220 \mathrm{~K}$ which required an individual amplitude $A$ for a satisfactory fit. ${ }^{34}$ The values of the common parameters are shown in Table I. The standard deviation of the fitted parameters were estimated using a Monte Carlo method ${ }^{35}$ in which the model function is fitted to synthetic data sets. The synthetic data sets are generated from the fit to the original data and from the statistical errors in those data. The standard deviations for the parameters found in the fits to the synthetic data are readily calculated, and then assumed to be similar to those for the parameters from the fit to the original data. Recently, Mos et al. performed an NSE experiment on PEO and PEO-LiI O:Li=15:1 using the IN11 instrument (incoherent scattering) and reported a similar decrease of the stretching parameter $\beta$ from about 0.57 for the pure polymer to 0.44 for the polymer salt complex. ${ }^{36}$ Mao et al. found a $\beta$ parameter of approximately 0.61 for PEO in an experiment performed on the IRIS instrument (incoherent scattering). ${ }^{37}$ These values are in accordance with our findings.

Regarding the fits of the IRIS data (Figs. 5 and 6) we have to note that $S(Q, t) / S(Q, 0)$ values with $t<3$ ps lie clearly above the KWW fit. This behavior is qualitatively expected because of the "picosecond process,", 38 but the crossover time is much larger than observed in other polymers, where it is $t \approx 1 \mathrm{ps}$. Methyl group motion can be ruled out as the source of this effect because it was just the methyl group hydrogen atoms that were nearly completely replaced by deuterium in our samples. ${ }^{39}$ This effect has been more carefully studied on an instrument which is more appropriate for this time range-the IN6 TOF spectrometer in Grenoble - and is the subject of a forthcoming paper.

Figure 7 shows the average relaxation time $\langle\tau\rangle$, i.e.,

$$
\langle\tau\rangle=\frac{\tau_{K}}{\beta} \Gamma\left(\frac{1}{\beta}\right)
$$

as obtained for the different sample/instrument combinations in the fitting procedure. Comparing the different samples, it is clear that the dynamics of the polymer salt complex is significantly slower than the pure polymer (see Fig. 7) and the increased broadening of the relaxation in the Li-doped sample is reflected in the smaller $\beta$ value (see Table I). On the other hand, the differences with respect to the instrument used are unexpected, because the quantity $S(Q, t) / S(Q, t$ $=0)$ should be defined in approximately the same way. The

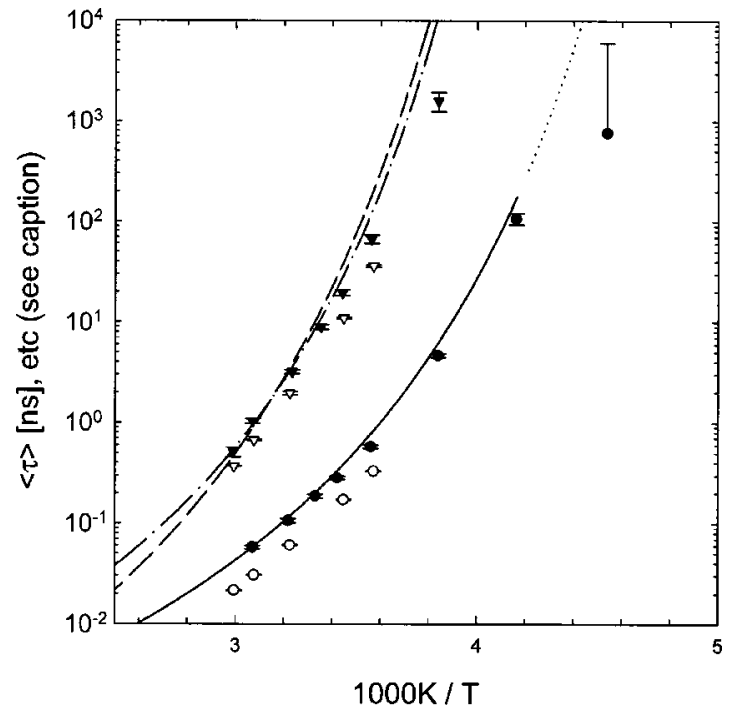

FIG. 7. Arrhenius plot of $\langle\tau\rangle$ for PPO (rings) and $\mathrm{PPO}-\mathrm{LiClO}_{4} \mathrm{O}: \mathrm{Li}$ $=16: 1$ (triangles) as obtained from fitting Eq. (2) to the data from the IRIS instrument (open symbols) and the IN11 instrument (filled symbols). The error bars denote the standard deviations estimated as described in the text. The lower part of the error bar for the $\langle\tau\rangle$ value for PPO at $T=220 \mathrm{~K}$ is omitted since it extends to negative values. Included for comparison are scaled temperature dependences of the viscosity for PPO: $\eta / 50 \mathrm{P}$ (solid line where the underlying data was measured and dotted line for extrapolation), for PPO-LiClO 4 O:Li=16:1: $\eta / 100$ Poise (dashed line) (see Refs. 42 and 43) and the scaled conductivity of PPO- $\mathrm{LiClO}_{4} \quad \mathrm{O}: \mathrm{Li}=16: 1$ : $2.5 \cdot 10^{-5} \mathrm{~S} \mathrm{~cm}^{-1} / \sigma$ (dashed-dotted line) (see Ref. 42).

smaller $\beta$ parameter for IRIS indicates that an additional broadening is observed. The additional broadening can be attributed to a larger fraction of incoherent scattering; investigations of hydrogenous PPO and PPO- $\mathrm{LiClO}_{4}$ on the IRIS spectrometer indicate a $\beta=0.41$ and $\beta=0.35$, respectively, and slightly different relaxation times. ${ }^{40}$ While on the NSE instrument incoherent scattering will only contribute with $1 / 3$ weight, no such discrimination between incoherent and coherent scattering is possible on the IRIS instrument. Because of the poor deuteration of the polymer the incoherent scattering amounts to about $41 \%$ even at the structure factor peak.

Similarly the smaller value of the amplitude factor $A$ may be attributed to an admixture of incoherent scattering. Here in addition an incorrect normalization due to the presence of a sample dependent background may play a role. The IRIS spectra shown in Figs. 1 and 2 clearly show the presence of such a background by the unphysical increase of $S(Q, \omega)$ for energy transfers $\hbar \omega>4 \mathrm{meV}$. Because this background increases with temperature it is difficult to estimate to what extent it affects the range $<2.5 \mathrm{meV}$ used in the Fourier transform and thereby the normalization of $S(Q, t) / S(Q, t=0)$.

Because of these obvious discrepancies of the relaxation shape observed on NSE and IRIS we refrained from splicing together the time-domain data. In order to do this arbitrary factors would have to be applied which cannot be justified on physical grounds. The data from the IN11 and the IRIS instruments will therefore be treated separately. 


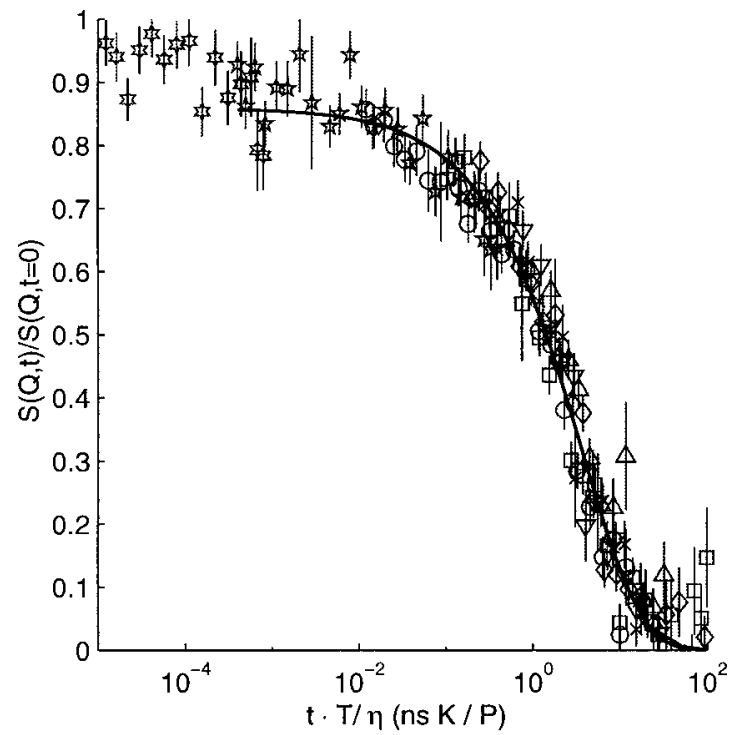

FIG. 8. $S(Q, t \cdot T / \eta(T)) / S(Q, 0)$ at $Q=1.45 \AA^{-1}$ for PPO as obtained from the NSE experiments [(stars) $220 \mathrm{~K}$, (plus) $240 \mathrm{~K}$, (rings) $260 \mathrm{~K}$, (squares) $280 \mathrm{~K}$, (diamonds) $290 \mathrm{~K}$, (crosses) $300 \mathrm{~K}$, (down triangle) $310 \mathrm{~K}$, and (up triangle) $325 \mathrm{~K}$ ]. Solid line represent a fit of a stretched exponential function [Eq. (2)] to the data points.

\section{DISCUSSION}

The results clearly show that the solvation of the salt in the polymer matrix induces large changes in the dynamics of the matrix, indicating a significant interaction between the ions and the polymer chains. To investigate the character of this interaction we focus on the molecular origin of the observed relaxation and compare it to the macroscopic viscosity. We scrutinize the explanation of the additional relaxation broadening in salt doped polymers by heterogeneity. Finally, we discuss the local motion of the ions within the framework of the DDH model using the characteristic relaxation times found for the polymer matrix and literature data on the ionic conductivity.

The momentum transfer $Q=1.45 \AA^{-1}$ investigated in the present experiments is approximately that of the FSDP in the static structure factor $S(Q)$. Neutron diffraction investigations of $\mathrm{PPO}^{26}$ and $\mathrm{PPO}-\mathrm{LiClO}_{4} \mathrm{O}: \mathrm{Li}=16: 1^{27}$ show that the FSDP originates mainly from interchain correlations, that is the correlation between chain segments belonging to distinctly different chains or segments of the same chain separated by a contour length larger than the persistence length. The relaxation behavior probed in the present study can therefore be attributed to the decay of the spatial correlation between neighboring chain segments. This is in agreement with findings for polybutadiene. ${ }^{41}$

\section{A. Salt induced effects on the polymer host}

To investigate whether the observed dynamics is the primary relaxation, i.e., the $\alpha$ relaxation commonly believed to be related to the segmental motion of the main chain in polymers, we scale the obtained relaxation functions with data of the viscosity $\eta$ found in the literature. ${ }^{42,43}$ More precisely, the $\alpha$ relaxation should - as justified by the Rouse modelscale with $\eta(T) / T{ }^{44}$ This kind of scaling relation has been found valid for several polymers, e.g., polybutadiene. ${ }^{45}$

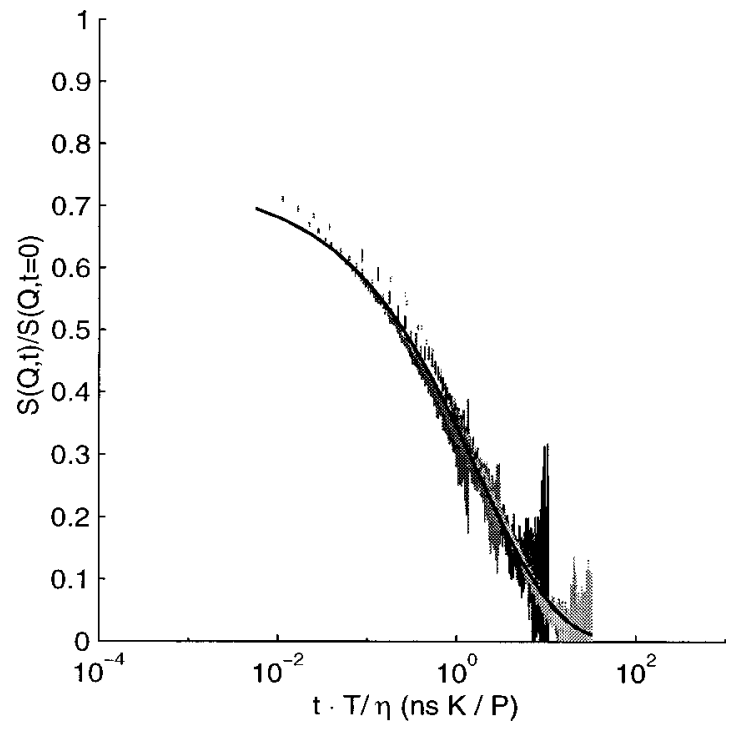

FIG. 9. $S(Q, t \cdot T / \eta(T)) / S(Q, 0)$ at $Q=1.45 \AA^{-1}$ for PPO as obtained on the IRIS instrument. The temperatures are from left to right 280, 290, 310, 325 , and $334 \mathrm{~K}$. Solid line represent a fit of a stretched exponential function [Eq. (2)] to the data points.

Figures 8 and 9 show the relaxation behavior for PPO where the experimental time $t$ is scaled by $\eta(T) / T$, i.e., $S(Q, t \cdot T / \eta(T)) / S(Q, 0)$.

The temperature dependencies of the viscosities were constructed after a comparison of the literature available on PPO of molecular weight 4000 and its 16:1 mixture with $\mathrm{LiClO}_{4}$. While the data of McLin and Angell ${ }^{42}$ agree well with those of Eisenberg et al., ${ }^{43}$ those of Wixwat et al. ${ }^{46}$ deviate significantly from the former. For PPO-LiClO 4 16:1 we therefore decided to use the viscosity values given in Refs. 42 and 43 together and fitted them by a VogelFulcher expression $\ln \left(\eta_{\mathrm{PPO}-\mathrm{LiClO}_{4}} / \mathrm{P}\right)=-5.8+1351 \mathrm{~K} /(T$ $-194.2 \mathrm{~K})$. This expression describes the data well over the whole range of 280-400 K. For pure PPO, raw viscosity data are not given in Ref. 42. Only the Vogel-Fulcher parameters $B=939 \mathrm{~K}$ and $T_{0}=174 \mathrm{~K}$ are stated. From the experimental details mentioned in Ref. 42 we estimate the range of validity for this expression to be $240-400 \mathrm{~K}$. Because this range is larger than the range used in Ref. 43 we fitted the latter data keeping the parameters $B$ and $T_{0}$ fixed and finally obtained $\ln \left(\eta_{\mathrm{PPO}} / \mathrm{P}\right)=-5.1+939 \mathrm{~K} /(T-174 \mathrm{~K})$. The conductivity data of Ref. 42 for $\mathrm{PPO}-\mathrm{LiClO}_{4}$ 16:1 in the range 260-410 K was analogously fitted by a Vogel-Fulcherlike expression $\ln \left(\sigma_{\mathrm{PPO}-\mathrm{LiClO}_{4}} / \mathrm{S} \mathrm{cm}^{-1}\right)=-1.8-1094 \mathrm{~K} /(T$ $-200.0 \mathrm{~K})$.

It can be seen for the pure polymer that the rescaled data points fall on master curves, except for the lowest temperature, $T=220 \mathrm{~K}$. (The scaling is not perfect but considering the difficulties obtaining reliable viscosity values we do not consider the deviations significant.) As expected the data points in the spectra for $T \geqslant 240 \mathrm{~K}$ are well described by Eq. (2) as demonstrated by the fits shown in Figs. 8 and 9. We therefore attribute the observed relaxation to the primary $\alpha$ process.

$S(Q, t \cdot T / \eta(T)) / S(Q, 0)$ for $\mathrm{PPO}-\mathrm{LiClO}_{4} \mathrm{O}: \mathrm{Li}=16: 1$ is shown in Figs. 10 and 11. It is seen that the relaxation ob- 


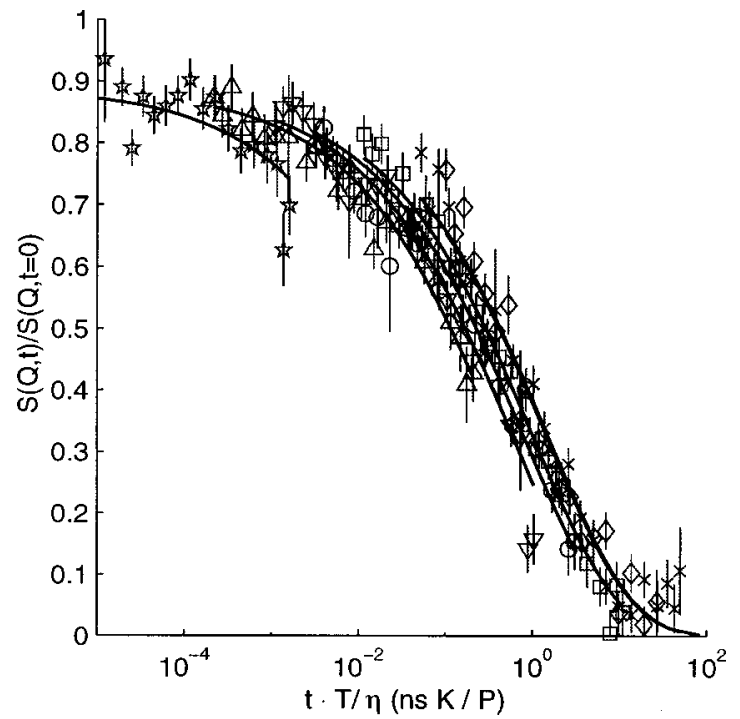

FIG. 10. $S(Q, t \cdot T / \eta(T)) / S(Q, 0)$ at $Q=1.45 \AA^{-1}$ for $\mathrm{PPO}-\mathrm{LiClO}_{4} \mathrm{O}: \mathrm{Li}$ $=16: 1$ as obtained from the NSE experiment [(stars) $260 \mathrm{~K}$, (up triangle) $280 \mathrm{~K}$, (down triangle) $290 \mathrm{~K}$, (rings) $300 \mathrm{~K}$, (squares) $310 \mathrm{~K}$, (crosses) 325 $\mathrm{K}$, and (diamonds) $334 \mathrm{~K}$ ]. Solid lines represent the fits of a stretched exponential function [Eq. (2)] with parameters in Table I to the data points.

served at the $Q$ value of the FSDP in $S(Q)$ does not scale with $\eta(T) / T$ as is the case for the pure polymer. ${ }^{47}$ Consequently, it may be argued that it is not the $\alpha$ relaxation that is observed here. On the other hand, neutron diffraction investigations ${ }^{27}$ of $\mathrm{PPO}-\mathrm{LiClO}_{4} \mathrm{O}: \mathrm{Li}=16: 1$ established that the FSDP originates from interchain correlations. Therefore, the molecular origin of the relaxation must be the same in as in the pure polymer. The failure of the viscosity scaling has to be ascribed to salt induced changes to the dynamics of the polymer matrix.

A plausible explanation may be based on the interaction between the polymer chains and the cations, which as they

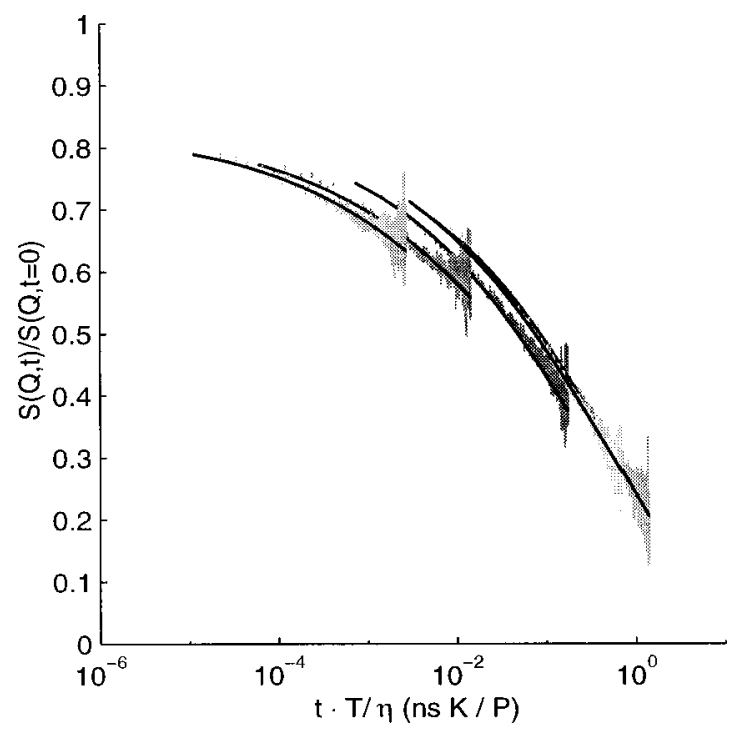

FIG. 11. $S(Q, t \cdot T / \eta(T)) / S(Q, 0)$ at $Q=1.45 \AA^{-1}$ for PPO- $\mathrm{LiClO}_{4} \mathrm{O}: \mathrm{Li}$ $=16: 1$ as obtained on the IRIS instrument. The temperatures are from left to right 280, 290, 310, 325, and $334 \mathrm{~K}$. Solid lines represent the fits of a stretched exponential function [Eq. (2)] with parameters in Table I to the data points. coordinate to the ether-oxygens in the polymer chains, may form cross links between chains and thereby change the effective molecular weight. The mechanism of transient cross linking has been suggested to cause the changes in the temperature dependence of the average relaxation time for polymer salt complexes compared to pure polymers. ${ }^{4}$ Since the number of dissociated ions, i.e., ions that can take part in a transient cross link, decrease with temperature, ${ }^{13}$ the effective molecular weight will be temperature dependent. Since the viscosity of a polymer depends on the molecular weight, whereas the $\alpha$ relaxation does not, the rescaling of the $\alpha$ relaxation with $\eta / T$ does not hold. A similar effect has been observed in supercooled selenium, in which the atoms form linear polymer chains of a length that depends on temperature. $^{48}$

In the data shown in Figs. 3-6 as well as the fit parameters in Fig. 7 and Table I the relaxation of PPO- $\mathrm{LiClO}_{4}$ is seen to be slower and more stretched compared to that of pure PPO. The slowing down is a generally observed phenomenon in polymer electrolytes and has been studied mainly by light scattering techniques (see for instance Refs. $5,17,49$, and 50) and recently also neutron spin echo. ${ }^{36,51}$ This can be explained by several possible ways of interaction between the polymer chains and the cations. Cations bound to several ether oxygen atoms of one polymer chain may induce a stiffening of the chain (intrachain effect). Cations bound to different chains may act as temporary cross links (interchain effect) raising $T_{g}$ with respect to the uncrosslinked polymer. This effect is generally observed for permanently cross linked polymers and may be explained in the framework of the Gibbs-DiMarzio theory. ${ }^{52}$

It is more difficult to understand why the observed stretching parameter $\beta$ is smaller in the salt doped polymer than in the pure polymer. The stretching is generally observed for polymer electrolytes. Indeed, even the effect of permanent cross links on the relaxation shape is still discussed in the literature with the majority of investigations showing an increase in broadening (e.g., Ref. 53) but some also an unchanged $\beta$ parameter. ${ }^{54}$ The additional stretching may suggest a broadening due to heterogeneity. Differential scanning calorimetry (DSC) experiments ${ }^{42}$ on PPO- $\mathrm{LiClO}_{4}$ $\mathrm{O}: \mathrm{Li}=16: 1$ show a broader glass transition. Another set of DSC experiments on PPO- $\mathrm{LiClO}_{4} \mathrm{O}: \mathrm{Li}=16: 1^{55,56}$ even show two glass transitions which are attributed to the extreme case of salt rich and approximately salt free regions of the polymer matrix. Reports on incoherent neutron scattering experiments ${ }^{40}$ show large deviations from the Gaussian approximation can also be interpreted as signatures of heterogeneity.

The present experiments measure the dynamics on length scales corresponding to the interchain distance. This means that in the case of a sample with heterogeneities, e.g., salt concentration fluctuations, which are larger in size than the interchain distance, we will observe a superposition of the relaxation functions from the different regions of the polymer (heterogeneities). Since the relaxation time $\tau$ increases with salt concentration, heterogeneities in terms of salt concentration fluctuations would lead to an observed relaxation, which can be described by a sum of relaxation 
functions with different relaxation times. Such a superposition of relaxations would be observed experimentally as an extra stretching (a lower $\beta$ value). The technique of using a superposition of relaxation functions of a pure polymer has in several instances been successfully used in the description of concentration fluctuations in polymer blends. ${ }^{57-59}$ Indeed, it is possible to fit the NSE and IRIS data of PPO- $-\mathrm{LiClO}_{4}$ by a distribution of KWW functions

$$
\frac{S(Q, t)}{S(Q, 0)}=A \int_{-\infty}^{+\infty} \exp \left(-\left(t / \tau_{K}\right)^{\beta}\right) G\left(\ln \tau_{K}\right) d \ln \tau_{K}
$$

with the respective $\beta$ value of pure PPO. If we chose the distribution of KWW times to be a log-normal

$$
G\left(\ln \tau_{K}\right)=\frac{1}{\sqrt{2 \pi} \sigma_{\ln \tau_{K}}} \exp \left(-\frac{\left(\ln \left(\tau_{K} / \tau_{0}\right)\right)^{2}}{2 \sigma_{\ln \tau_{K}}^{2}}\right)
$$

the resulting widths are $\sigma_{\ln \tau_{K}}=1.94$ for the NSE experiment and 2.40 for IRIS from joint fits of the spectra at different temperatures.

Of course the sole fact that such a fit is possible does not prove the presence of heterogeneity. In fact there is a strong argument that heterogeneity-if present at all-is not the only cause for stronger broadening in the salt doped system. Heterogeneity would, as discussed above, give a relaxation function which is a superposition of relaxation functions with different relaxation times. The relation between logarithms of relaxation times $\log \tau$ (e.g., as displayed in Fig. 7) for regions with different salt concentrations increases with decreasing temperature. Because of the increasing difference between the relaxation times at lower temperatures, cuts at constant temperature yield broader distributions of $\log \tau$ for lower temperatures. So the fact that $S(Q, t) / S(Q, 0)$ data exhibit the same stretching for all temperatures contradicts heterogeneity as the origin of the broadening.

In order to put this argument in a more quantitative form and to test whether our experiments are sensitive enough to detect differences in the broadening, we introduce a model for the heterogeneity. From the literature data of the viscosity $^{42,43}$ we conclude that the influence of the ions can be described as a rescaling of temperature ${ }^{60}$

$$
\left.\tau_{\mathrm{PPO}-\mathrm{LiClO}_{4}}(T)=\tau_{\mathrm{PPO}\left(x\left(c_{\mathrm{LiClO}_{4}}\right)\right.} \cdot T\right) .
$$

We can fit the experimental data of $\mathrm{PPO}-\mathrm{LiClO}_{4}$ with this expression and $x(16: 1)=0.8381$ nearly as well as with the free Vogel-Fulcher fit used above.

Even without knowing the functional dependence of $x$ on the ion concentration we can estimate its fluctuation from DSC experiments: ${ }^{42}$ The width of the glass transition is stated as $4 \mathrm{~K}$ for pure $\mathrm{PPO}$ and $11 \mathrm{~K}$ for $\mathrm{PPO}-\mathrm{LiClO}_{4}$. In order to convert these width values to variances we used the relation $\sigma_{T}^{2}=\Delta T^{2} / 12$ valid for a uniform distribution yielding $1.3 \mathrm{~K}^{2}$ for pure PPO and $10.1 \mathrm{~K}^{2}$ for PPO- $\mathrm{LiClO}_{4}$. Because the variance is additive in a convolution the additional width of the glass transition possibly arising from heterogeneity corresponds to a variance of the glass transition temperature of $8.8 \mathrm{~K}^{2}$ or a standard deviation $\sigma_{T}=3.0 \mathrm{~K}$. Because the center of the glass transition has been found at 229

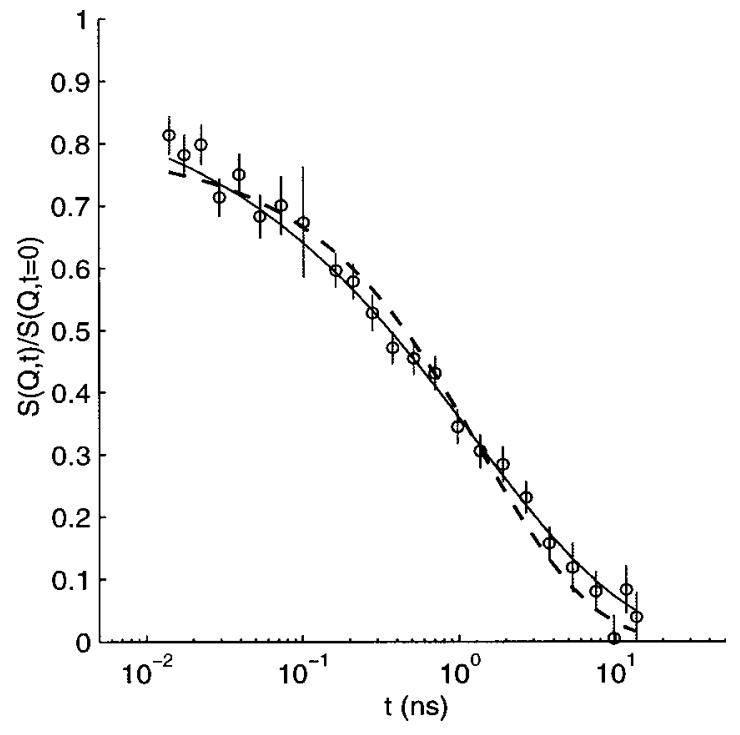

FIG. 12. NSE $S(Q, t) / S(Q, 0)$ data measured at $T=310 \mathrm{~K}$ and fitted with a superposition of KWW functions, Eq. (4), with $\beta=0.67$ from pure PPO and a log-normal distribution, Eq. (5), of characteristic times $\tau_{K}$ forcing $\sigma_{\ln } \tau_{K}$ $=0.62$ (dashed line). Alternatively, the usual KWW fit, Eq. (2), is shown (continuous line).

$\mathrm{K}$ this corresponds to a standard deviation $\sigma_{x}=\sigma_{T} / T$ $=0.013$ of the temperature rescaling factor $x$.

Assuming that this value does not change strongly with temperature we use it together with Eq. (6) to estimate the standard deviation of the distribution of $\ln \tau_{K}$. By using the Vogel-Fulcher expression in Eq. (6) and taking the derivative with respect to $x$ we obtain

$$
\sigma_{\ln \tau_{K}}=\frac{B T}{\left(x T-T_{0}\right)^{2}} \sigma_{x} .
$$

With the values $B=939 \mathrm{~K}$ and $T_{0}=174 \mathrm{~K}$ for pure PPO, this expression yields a value $\sigma_{\ln \tau_{K}}=1.66$ at $260 \mathrm{~K}$ decreasing to $\sigma_{\ln \tau_{K}}=0.366$ at $334 \mathrm{~K}$. The strong temperature dependence is plausible because $260 \mathrm{~K}$ is already close to the glass transition temperature $(229 \mathrm{~K})$.

For a quantitative check in Fig. 12 we show NSE data at $T=300 \mathrm{~K}$ fitted with Eqs. (4) and (5) using $\sigma_{\ln \tau_{K}}=0.62$ from Eq. (7) and alternatively the KWW fit. It can be seen that both fits follow the data points roughly within their error bars. Nevertheless, the distribution fit is not sufficiently "broadened" as indicated by the systematic deviations at short and long times. In addition it results in a prefactor $A$ $=0.79$ which is too low.

Other procedures may be devised to compare the broadening of the glass transition in DSC experiments to that of the relaxation function. It is, however, clear that the broadening $\sigma_{\ln \tau_{K}}$, together with the relaxation function of pure PPO in Eqs. (4)-(7) does not give a relaxation function which is sufficiently stretched to describe the present data of PPO- $\mathrm{LiClO}_{4}$ 16:1. Therefore, the broadening of the relaxation must be (at least to its major part) of intrinsic nature, and is not the result of a distribution of correlation times arising from heterogeneities induced by the ions. 
It has to be conceded that there remains the possibility of heterogeneity on a microscopic level. Because inelastic neutron scattering observes the motions on atomic length scales it is sufficient that some of the scattering atoms move faster than others to create a broadened spectrum. It is not a priori clear what the size of the regions is which determine the glass transition temperature in DSC experiments. However, the microheterogeneous picture would strongly resemble polymer blends of polystyrene/poly(vinyl methyl ether). ${ }^{59}$ For such blends, DSC experiments show a single glass transition unless the blend macroscopically demixes. ${ }^{61}$ Nevertheless, the microscopic dynamics seen by quasielastic neutron scattering reveals that the component polymers have strongly different relaxation times. We can thereby assume that the regions probed by glass transition determinations using the DSC method are larger than the length scale of the neutron scattering experiments presented here. Therefore a microscopic heterogeneity may be present which is partially averaged out in the DSC experiment. If this extra heterogeneity exists and would be detected by the DSC experiment, it could be included in our fitting procedure above and it may be possible that a distribution of relaxation times based on the broadening by DSC would fit the NSE data.

Also the fact that all spectra taken at different temperatures from the NSE or IRIS experiments on PPO- $\mathrm{LiClO}_{4}$ can be fitted with the same width $\sigma_{\ln \tau}$ (or $\beta$ in the KWW fits) could at first glance be considered a strong argument against a heterogeneous nature of the broadening in the polymer salt system. In general, one would expect an increasing broadening at low temperature for a heterogeneous system from a relation as, e.g., Eq. (7). The range of 3-4 decades in time covered in our experiments is unfortunately not sufficient to determine broadening parameters as $\sigma_{\ln \tau}$ or $\beta$ for each individual temperature. The experimental finding of constant broadening parameter must therefore be restricted to apply only to the time range covered by the experiments.

The results presented show that, in the samples investigated, there are no indications of inhomogeneous dynamics induced by the ions. It should be noted though, that for several polymer electrolytes including PPO- $\mathrm{LiCLO}_{4}$, experimental observations of two glass transitions are reported and interpreted as phase separation into salt rich and approximately salt free regions. ${ }^{55,56,62} \mathrm{We}$ note that these experiments were performed with DSC, and therefore probe the material properties largely in a lower temperature range than the present experiments. A possible explanation for this discrepancy between experiments is a degree of heterogeneity which decreases at higher temperatures. There are furthermore indications of a microscopic phase separation into salt rich and salt depleted regions which is dependent on thermal history. ${ }^{63}$

\section{B. Results on the DDH model}

Finally, we want to point out the relevance of neutron scattering data for the DDH model ${ }^{24}$ of the conductivity in polymer electrolytes. In this model the charge carriers are assumed to move in a disordered surrounding with their mean-square displacement following some functional form $\left\langle r^{2}(t)\right\rangle=g(t)$ until a renewal event occurs which restruc- tures the matrix. In that case the carrier motion is reinitialized, e.g., if a renewal happens at $t_{1}$ the mean-square displacement will follow $\left\langle r^{2}(t)\right\rangle=g\left(t_{1}\right)+g\left(t-t_{1}\right)$ for $t>t_{1}$. The central quantity of this model is the renewal time $\tau_{R}$, the average time between two subsequent renewal events.

In the earlier DBP model ${ }^{21}$ the elementary motion described by $g(t)$ was identified with a hopping motion on a lattice with a random arrangement of closed and open bonds. Although this specific assignment has been abandoned later in the DDH model the assumption was retained that in polymer electrolytes $g(t)$ would reach a limiting value $\left\langle r^{2}(\infty)\right\rangle$ if renewal events were absent. In the DBP this corresponds to the case of a system that would be below the percolation threshold in the absence of renewal.

In addition it is assumed that this saturation value is reached on a time scale much faster than that of the renewal process, $\tau_{R}$. Under these assumptions an additional Debye response should show up in conductivity spectra corresponding to the temporary restricted motion of the ions between the renewal events. Indeed, measurements of the frequency dependent conductivity ${ }^{64}$ show this signature lending support to the validity of the two assumptions.

In such a renewal controlled system below the percolation threshold the static diffusion coefficient assumes a simple form, regardless of the specific $g(t)$

$$
D(0)=\frac{\left\langle r^{2}(\infty)\right\rangle}{6 \tau_{R}} .
$$

In order to calculate the limiting mean-square displacement $\left\langle r^{2}(\infty)\right\rangle$ knowledge of $D(0)$ and $\tau_{R}$ is required. Because direct measurements of the diffusion coefficients in polymer electrolytes are few, it is usually expressed by the conductivity $\sigma$ via the Nernst-Einstein relation leading to

$$
\left\langle r^{2}(\infty)\right\rangle=\frac{6 \sigma \tau_{R} k_{B} T}{n_{\text {ions }} q^{2}} .
$$

Here, $n_{\text {ions }}$ denotes the carrier density taken from a Raman scattering study ${ }^{65}$ and $q$ their charge. However it should be noted that there are indications of that the Nernst-Einstein relation may be quantitatively invalid in polymer electrolytes. $^{7}$

The renewal time $\tau_{R}$ is identified with the $\alpha$ (or glass transition) relaxation time of the polymer matrix. Because the $\alpha$ relaxation is a ubiquitous phenomenon this leaves the choice open of which experimental method to use to determine $\tau_{\alpha}$. Previously, the dielectric relaxation time of the pure polymer was used and rescaled by the known shift of $T_{g}$ induced by the dissolved salt. ${ }^{24}$

Here, we propose that the average relaxation time $\langle\tau\rangle$ [Eq. (3)] determined by coherent neutron scattering at the structure factor peak may be a better choice. First, it describes the $\alpha$ relaxation on a microscopic length scale corresponding to the interchain distance. It is this kind of motions which should give rise to the renewal events. Second, it can be determined directly from the salt doped samples removing the uncertainty involved in the abovementioned rescaling process.

Figure 13 shows the values $\left\langle r^{2}(\infty)\right\rangle^{1 / 2}$ of the limiting distances obtained in this way. We note that most of the 


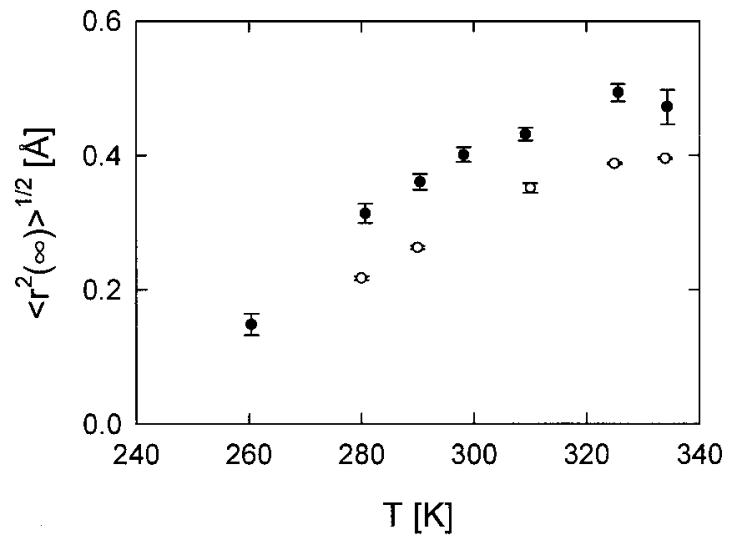

FIG. 13. Square root of the limiting mean-square displacement calculated with the dynamic disordered hopping model using the average relaxation time from coherent scattering at $Q=1.45 \AA^{-1}$. Filled circles refer to the NSE experiment empty circles to that on IRIS.

values are in the range of those reported earlier ${ }^{24}$ for other polymer electrolyte systems, $0.3-0.5 \AA$. The values derived from IRIS data are lower by about $30 \%$, which is a consequence of the lower relaxation times determined on this spectrometer.

The fact that our calculations reproduces the results of Lonergan et $_{\text {al. }}{ }^{24}$ supports the validity of their rescaling procedure and in turn their discussion of the magnitude of $\left\langle r^{2}(\infty)\right\rangle^{1 / 2}$. On the other hand, a significant temperature dependence remains in the values of $\left\langle r^{2}(\infty)\right\rangle^{1 / 2}$. Although it is weak if compared to the temperature dependences of the original $\sigma$ or $\langle\tau\rangle$ data it is still stronger than would be expected, e.g., from a vibrational mean-square displacement $\left\langle r^{2}(\infty)\right\rangle \propto T$.

As the error bars in Fig. 13 show, this temperature dependence is significant with respect to possible errors from the fits determining the average relaxation time from the neutron scattering data. On the other hand there would have to be an error of $150 \%$ in the conductivity data to explain the difference of $\left\langle r^{2}(\infty)\right\rangle^{1 / 2}$ between 280 and $325 \mathrm{~K} .{ }^{66}$ The deuteration of the PPO used here may cause a shift in $T_{g}$, which also could lead to an artificial temperature dependence. But DSC experiments on other polymers ${ }^{45}$ have shown that $T_{g}$ is higher in the deuterated polymer. In order to compensate the temperature dependence here, a negative shift of $T_{g}$ by about $-7 \mathrm{~K}$ would be necessary. Finally, the $\mathrm{LiClO}_{4}$ content would have to be wrong by more than $15 \%$ here or in the conductivity measurement which can also be excluded.

Thus we consider the temperature dependence of $\left\langle r^{2}(\infty)\right\rangle^{1 / 2}$ to be significant and rather ascribe it to immanent problems of its derivation. For instance, the Nernst-Einstein relation is used despite doubts concerning its validity for polymer electrolytes. ${ }^{7}$ Therefore, a crucial test of the DDH model would require a direct determination of the diffusion constant, e.g., by pulsed field gradient NMR.

\section{CONCLUSION}

We present the first investigation of the structural dynamics of a polymer electrolyte by coherent quasielastic scattering combining the NSE technique and inverse TOF spectroscopy. In order to discuss comparable quantities we Fourier transformed the TOF data obtaining a time range extending to more than 1 decade shorter times than those from NSE.

A direct combination of the data from both methods in the time domain was not possible because the incoherent scattering resulting from incomplete deuteration of the samples enters with different weight in the two experiments. For both instruments the intermediate scattering function $S(Q, t) / S(Q, t=0)$ can be described by a stretched exponential (KWW) function Eq. (2). The stretching parameter $\beta$ is smaller for the TOF data as a result of the incoherent contribution which is more stretched ${ }^{40}$ and has a different relaxation time.

Apart from this difference, data from both instruments show similar effects of the ions of the dissolved salt on the segmental motion of the polymer chains: (1) The $\alpha$ relaxation is slower in the salt-containing sample. (2) The $\alpha$ relaxation is broadened in the salt-containing sample. (3) While for pure PPO the intermediate scattering function could be rescaled to a master curve using viscosity data this was not possible in case of the salt-complexed PPO.

The slowing down of the $\alpha$ relaxation is a generally observed effect of solvation of salt in a polymer. It can be understood on grounds of the cations acting as temporary cross links raising the glass transition temperature. In addition the cations may bind to several ether oxygens of the same chain and thus increase its stiffness.

Concerning the broadening we have attempted to interpret the data in a heterogeneous picture of regions with different relaxation times but the same stretching parameter as in pure PPO. Although this model allows the description of both the NSE and TOF data, the width of the distribution of relaxation times does not correspond to the broadening of the glass transition. The spread of the glass transition actually observed by $\mathrm{DSC}^{42}$ is much too small to be the sole origin of the required broadening.

Therefore, the broadening must have a homogeneous (intrinsic) origin or result from dynamic heterogeneity on a short length scale. In the former case the relation between the broadening of the DSC glass transition and the relaxation observed by quasielastic neutron scattering is not a priori clear. The other possibility of microheterogeneity seems plausible because individual segments of the polymer chain may differ in mobility according to the way they interact with cations. Nevertheless, on the length scale of regions determining the glass transition temperature the heterogeneity may partially average out, leading to the smaller signature of heterogeneity in the DSC experiment. A distinction between these two sources cannot be done on grounds of the present data but would require, e.g., measuring the $Q$ dependence of the incoherent quasielastic scattering.

In passing we note that the smaller stretching parameter $\beta$ for the salt doped samples cannot be related to the fragility $^{67}$ in the framework of the coupling model. ${ }^{68}$ If a difference in fragility can be detected at all, $\mathrm{PPO}-\mathrm{LiClO}_{4}$ $\mathrm{O}: \mathrm{Li}=16: 1$ is a "stronger" liquid than pure $\mathrm{PPO}^{42}$ and therefore should have a higher $\beta$ parameter according to the coupling model. 
With respect to the scaling of the intermediate scattering function with a viscosity time $\tau_{\eta} \propto \eta / T$ which is usually seen as the signature of $\alpha$ relaxation "universality," 45 the samples show certain differences: For pure PPO the scaling is possible with the exception of the lowest temperature 220 $\mathrm{K}$. This temperature is below $1.2 \cdot T_{g}$, i.e., in a range where a decoupling of viscosity often is observed (see, e.g., Ref. 69). In contrast, for PPO- $\mathrm{LiClO}_{4}$ a deviation of about 1 decade occurs over the temperature range 280-334 K. Because this difference does not occur at a certain threshold temperature but rather builds up gradually when changing the temperature, it can be suspected that the coupling factor between microscopic friction and the viscosity depends on temperature here. A possible reason for such a temperature dependence would be that the temporary cross linking (and end linking because the PPO used here was hydroxy capped) by the cations varies with temperature as shown by Raman spectroscopy experiments. ${ }^{65}$ Thus, the effective molecular weight determining the relation between segmental $(\alpha)$ and terminal relaxation (viscosity) will not be constant, but vary with temperature.

Finally, we were able to use the microscopic average relaxation time from neutron scattering in the framework of the DDH model ${ }^{24}$ as the renewal time $\tau_{R}$. By doing this the uncertainty of using temperature rescaled data from dielectric loss maxima in the pure polymer can be removed. The values obtained for the limiting root-mean-square displacement $\left\langle r^{2}(\infty)\right\rangle^{1 / 2}$ are in remarkable agreement with those derived on other polymer electrolyte systems with the above mentioned assumptions. ${ }^{24}$ On the other hand, the fact that $\left\langle r^{2}(\infty)\right\rangle^{1 / 2}$ shows a significant and comparatively strong temperature dependence sheds some doubt on the validity of the DDH model or at least the use of the Nernst-Einstein equation in its context. ${ }^{70-72}$

\section{ACKNOWLEDGMENTS}

This work has benefitted greatly from suggestions and many valuable discussions with Professor P. Jacobsson and Professor D. Richter. Colleagues affiliated at Chalmers University of Technology would like to acknowledge financial support from the Swedish Natural Science Research Council. R.Z. gratefully acknowledges financial support from the Materials Consortium at Chalmers University of Technology.

${ }^{1}$ E. E. Fenton, J. M. Parker, and P. V. Wright, Polymer 14, 589 (1973).

${ }^{2}$ M. B. Armand, J. M. Chabagno, and M. J. Duclot, Extended abstracts, Second International Conference on Solid Electrolytes, St. Andrews, Scotland, 1978.

${ }^{3}$ C. Berthier, W. Gorecki, M. Minier, M. B. Armand, J. M. Chabgno, and P. Rigaud, Solid State Ionics 11, 91 (1983).

${ }^{4}$ L. M. Torell, in Handbook of Solid State Batteries and Capacitors, edited by M. Z. Z. Munshi and P. S. S. Prasad (World Scientific, Singapore, 1995), p. 311.

${ }^{5}$ F. M. Gray, Solid Polymer Electrolytes, Fundamentals and Technological Applications (VCH, New York, 1991).

${ }^{6}$ F. M. Gray, Polymer Electrolytes, Fundamentals and Technological Applications (The Royal Society of Chemistry, Cambridge, UK, 1997).

${ }^{7}$ V. A. Payne, M. C. Lonergan, M. Forsyth, M. A. Ratner, D. F. Shriver, S. W. de Leeuw, and J. W. Perram, Solid State Ionics 31, 171 (1995).

${ }^{8}$ P. Lightfoot, M. A. Mehta, and P. G. Bruce, Science 262, 883 (1993).

${ }^{9}$ G. S. MacGlashan, Y. G. Andreev, and P. G. Bruce, Nature (London) 398, 792 (1999).
${ }^{10}$ J. D. Londono, B. K. Annis, A. Habenschuss, O. Borodin, G. D. Smith, J. Z. Turner, and A. K. Soper, Macromolecules 30, 7151 (1997).

${ }^{11}$ O. Borodin and G. D. Smith, Macromolecules 31, 8396 (1998).

${ }^{12}$ L. M. Torell and S. Schantz, in Polymer Electrolyte Reviews, edited by J. R. MacCallum and C. A. Vincent (Elsevier, New York, 1989), Vol. 2, pp. $1-43$.

${ }^{13}$ S. Schantz, J. Chem. Phys. 94, 6296 (1991).

${ }^{14}$ A. Ferry, P. Jacobsson, and L. M. Torell, Electrochim. Acta 40, 2369 (1995).

${ }^{15}$ A. Ferry, J. Phys. Chem. B 101, 150 (1997).

${ }^{16}$ Polymers: Applications of Electroactive Polymers, edited by B. Scrosati (Chapman \& Hall, London, UK, 1993).

${ }^{17}$ L. M. Torell and C. A. Angell, Br. Polym. J. 20, 173 (1988).

${ }^{18}$ J. Sandahl, S. Schantz, L. Börjesson, L. M. Torell, and J. R. Stevens, J. Chem. Phys. 91, 655 (1989).

${ }^{19}$ M. A. Ratner, in Polymer Electrolyte Reviews, edited by J. R. MacCallum and C. A. Vincent (Elsevier, London, 1987), Vol. 1, Chap. 7.

${ }^{20}$ M. A. Ratner, MRS Bull. 14, 39 (1989).

${ }^{21}$ S. D. Druger, A. Nitzan, and M. A. Ratner, J. Chem. Phys. 79, 3133 (1983).

${ }^{22}$ M. A. Ratner and D. F. Shriver, Chem. Rev. 88, 109 (1988).

${ }^{23}$ A. Nitzan and M. A. Ratner, J. Phys. Chem. 98, 1765 (1994).

${ }^{24}$ M. C. Lonergan, A. Nitzan, M. A. Ratner, and D. F. Shriver, J. Chem. Phys. 103, 3253 (1995).

${ }^{25}$ Preliminary results from the NSE experiment were presented at the Eighth Tohwa University International Symposium 1998.

${ }^{26}$ P. Carlsson, J. Swenson, L. Börjesson, L. M. Torell, R. L. McGreevy, and W. S. Howells, J. Chem. Phys. 109, 8719 (1998).

${ }^{27}$ P. Carlsson, J. Swenson, D. Andersson, L. Börjesson, L. M. Torell, R. L. McGreevy, and W. S. Howells (unpublished).

${ }^{28}$ J. Lausmaa, Analysis report, Chemistry and Materials Technology, Swedish National Testing and Research Institute (unpublished).

${ }^{29}$ J. Allgaier (private communication).

${ }^{30}$ Due to noise in the data a strict upper limit of the acetonitrile concentration can be set to 0.002 acetonitrile molecules per repeat unit.

${ }^{31}$ Neutron Spin Echo: Lecture Notes in Physics, edited by F. Mezei (Springer, Heidelberg, 1980), Vol. 28.

${ }^{32}$ C. J. Carlile and M. A. Adams, Physica B 182, 431 (1992).

${ }^{33}$ W. S. Howells, IDA-Iris data analysis, 1996.

${ }^{34}$ This may be understood in the framework of the Mode Coupling Theory which predicts an increase of the $A$ parameter below the critical temperature $T_{c}$, which for PPO is approximately $236 \mathrm{~K}$.

${ }^{35}$ W. H. Press, B. P. Flannery, S. A. Teukolsky, and W. T. Vetterling, Numerical Recipes, The Art of Scientific Computing (Cambridge University Press, Cambridge, UK, 1986).

${ }^{36}$ B. Mos, P. Verkerk, S. Pouget, A. van Zon, G.-J. Bel, S. W. de Leeuw, and C. D. Eisenbach, J. Chem. Phys. 113, 4 (2000).

${ }^{37}$ G. Mao, R. Fernandez Perea, W. S. Howells, D. L. Price, and M.-L. Saboungi, Nature (London) 405, 163 (2000)

${ }^{38}$ R. Zorn, A. Arbe, J. Colmenero, B. Frick, D. Richter, and U. Buchenau, Phys. Rev. E 52, 781 (1995).

${ }^{39}$ Because of the pronounced threefold minimum potential for the rotational angle of the methyl group it is expected that this motion consists of jumps between three equivalent positions. Because of this equivalence the motion is only visible in incoherent scattering, which is strongly reduced by the deuteration. Experimentally, this expectation has been confirmed on samples of polyisoprene with different partial deuteration.

${ }^{40}$ D. Andersson, P. Carlsson, D. Engberg, L. M. Torell, L. Börjesson, R. L. McGreevy, and W. S. Howells, Physica B 266, 126 (1999).

${ }^{41}$ A. Arbe, D. Richter, J. Colmenero, and B. Farago, Phys. Rev. E 54, 3853 (1996).

${ }^{42}$ M. G. McLin and C. A. Angell, J. Phys. Chem. 95, 9464 (1991).

${ }^{43}$ A. Eisenberg, K. Ovans, and H. N. Yoon, in Advances in Chemistry Series 187: Ions in Polymers, edited by A. Eisenberg (American Chemical Society, Washington, DC, 1980), pp. 267-282.

${ }^{44}$ J. D. Ferry, Viscoelastic Properties of Polymers (Wiley, New York, 1980).

${ }^{45}$ D. Richter, B. Frick, and B. Farago, Phys. Rev. Lett. 61, 2465 (1988).

${ }^{46}$ W. Wixwat, Y. Fu, and J. R. Stevens, Polymer 32, 1181 (1991).

${ }^{47}$ As a cautious remark we have to note that the deviation from scaling is smaller if the deviating viscosity data from Ref. 46 are used.

${ }^{48}$ Ch. Simon, G. Faivre, R. Zorn, F. Batallan, and J. F. Legrand, J. Phys. I 2 , 307 (1992).

${ }^{49}$ J. Moacanin and E. F. Cuddihy, J. Appl. Polym. Sci. 14, 313 (1966).

${ }^{50}$ L. M. Torell and S. Schantz, J. Non-Cryst. Solids 131, 981 (1991). 
${ }^{51}$ P. Carlsson, R. Zorn, D. Andersson, D. Richter, L. M. Torell, L. Börjesson, P. Jacobsson, and B. Farago, in Slow Dynamics in Complex Systems: Eighth Tohwa University International Symposium, edited by M. Tokuyama and I. Oppenheim (American Institute of Physics, Melville, NY, 1999).

${ }^{52}$ E. A. DiMarzio, J. Res. Natl. Bur. Stand., Sect. A 68, 611 (1964).

${ }^{53}$ C. M. Roland, Macromolecules 27, 4242 (1994).

${ }^{54}$ B. D. Fitz and J. Mijovic, Macromolecules 32, 3518 (1999).

${ }^{55}$ C. Vachon, M. Vasco, M. Perrier, and J. Prud'homme, Macromolecules 26, 4023 (1993).

${ }^{56}$ C. Vachon, C. Labréche, A. Vallée, S. Besner, M. Dumont, and J. Prud'homme, Macromolecules 28, 5585 (1995).

${ }^{57}$ A. Zetsche and E. W. Fischer, Acta Polym. 45, 168 (1994).

${ }^{58}$ G. Katana, E. W. Fischer, T. Hack, V. Abetz, and F. Kremer, Macromolecules 28, 2714 (1995).

${ }^{59}$ I. Cendoya, A. Alegría, J. M. Alberdi, J. Colmenero, H. Grimm, D. Richter, and B. Frick, Macromolecules 32, 4065 (1999).

${ }^{60}$ Cendoya alternatively propose a change in $T_{0}$ at constant $B$ induced by concentration fluctuations in a polymer blend. In contrast, the rescaling by Eq. 6 is equivalent to a change of $T_{0}$ at constant fragility parameter $D$ $=B / T_{0}$. The two alternatives are difficult to distinguish over a limited temperature range but in our case the second one gives slightly better agreement.
${ }^{61}$ A. Zetsche, F. Kremer, W. Jung, and H. Schulze, Polymer 31, 1883 (1990).

${ }^{62}$ M. Bégin, C. Vachon, C. Labréche, B. Goulet, and J. Prud'homme, Macromolecules 31, 96 (1998).

${ }^{63}$ M. Furlani (unpublished).

${ }^{64}$ T. Furukawa, M. Imura, and H. Yuruzume, Jpn. J. Appl. Phys., Part 1 36, 1119 (1997).

${ }^{65}$ S. Schantz, L. M. Torell, and J. R. Stevens, J. Chem. Phys. 94, 6862 (1991).

${ }^{66}$ Not much of the relaxation is within the experimental window at $260 \mathrm{~K}$, and we therefore exclude this possibly unreliable point.

${ }^{67}$ C. A. Angell, in Relaxation in Complex Systems, edited by K. L. Ngai and G. B. Wright (National Technical Information Service, Springfield, VA, 1985), p. 3.

${ }^{68}$ K. L. Ngai, R. W. Rendell, and D. J. Plazek, J. Chem. Phys. 94, 3018 (1991).

${ }^{69}$ D. Richter, R. Zorn, B. Farago, B. Frick, and L. J. Fetters, Phys. Rev. Lett. 68, 71 (1992).

${ }^{70}$ W. Götze, J. Phys.: Condens. Matter 11, 41 (1999).

${ }^{71}$ D. Sidebottom, R. Bergman, L. Börjesson, and L. M. Torell, Phys. Rev. Lett. 68, 3587 (1992).

${ }^{72}$ B. Frick and L. J. Fetters, Macromolecules 27, 974 (1994). 